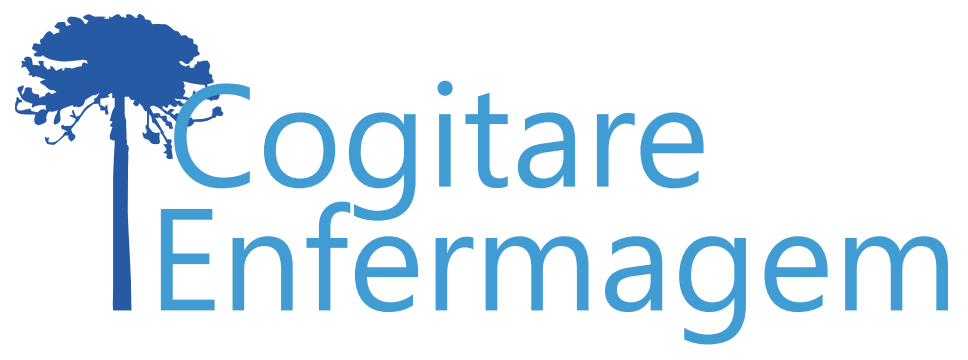

\title{
FATORES ASSOCIADOS À INDEPENDÊNCIA FUNCIONAL DE IDOSOS LONGEVOS DA COMUNIDADE
}

\author{
Darlene Mara dos Santos Tavares ${ }^{1}$, Nayara Cândida Gomes², Lara Arruda Lacerda Soares ${ }^{3}$, \\ Gianna Fiori Marchiori ${ }^{4}$
}

\section{RESUMO}

Objetivos: verificar a frequência de independência funcional entre os longevos e identificar os fatores associados à independência funcional dos longevos.

Metodologia: estudo transversal com 174 idosos de 80 anos e mais, residentes na zona urbana em Minas Gerais no período de junho de 2017 até junho de 2018. Para a seleção da população, utilizouse a amostragem por conglomerado em múltiplo estágio. Procederam-se às análises descritiva, bivariada e regressão logística múltipla $(p \leq 0,05)$.

Resultados: eram do sexo feminino $67,8 \%$, com cinco ou mais morbidades autorreferidas $62,6 \%$, $74,7 \%$ sem sintomas depressivos e $85,6 \%$ eram independentes nas atividades básicas da vida diária. A independência funcional nas atividades básicas da vida diária foi ausência de indicativo de sintomas depressivos $(p=0,046)$ e as atividades instrumentais da vida diária ao arranjo de moradia $(p=0,045)$.

Conclusão: a identificação dos fatores associados à independência funcional dos longevos auxiliam o planejamento do cuidado de enfermagem com vistas a diminuir ou postergar a dependência.

DESCRITORES: Idoso de 80 anos ou mais; Longevidade; Atividades Cotidianas; Enfermagem Geriátrica.

COMO REFERENCIAR ESTE ARTIGO:

Tavares DM dos S, Gomes NC, Soares LAL, Marchiori GF. Fatores associados à independência funcional de idosos longevos da comunidade. Cogitare enferm. [Internet]. 2019 [acesso em "colocar data de acesso, dia, mês abreviado e ano"]; 24. Disponível em: http://dx.doi.org/10.5380/ce.v24i0.61527.

\section{(c) (1)}

Este obra está licenciado com uma Licença Creative Commons Atribuição 4.0 Internacional.

${ }^{1}$ Enfermeira. Doutora em Enfermagem. Docente de Enfermagem da Universidade Federal do Triângulo Mineiro. Uberaba, MG, Brasil.

${ }^{2}$ Enfermeira. Doutorando em Atenção à Saúde. Universidade Federal do Triângulo Mineiro. Uberaba, MG, Brasil. $\odot$

${ }^{3}$ Discente de Enfermagem. Universidade Federal do Triângulo Mineiro. Uberaba, MG, Brasil. $(0)$

${ }^{4}$ Enfermeira. Doutorando em Atenção à Saúde. Universidade Federal do Triângulo Mineiro. Uberaba, MG, Brasil. $\bigcirc$ 


\title{
FACTORS ASSOCIATED WITH THE FUNCTIONAL INDEPENDENCE OFCOMMUNITY-DWELLING ELDERLY INDIVIDUALS AGED 80 OR OVER
}

\begin{abstract}
Objectives: To verify the frequency of functional independence among elderly people aged 80 or over and identify the factors associated with their functional independence.

Methodology: Cross-sectional study with 174 elderly people aged 80 or over living in the urban area of Minas Gerais conducted from June 2017 to June 2018. Multistage cluster sampling was used for the selection of the population. Descriptive, bivariate and multiple logistic regression analyzes were performed ( $p \leq 0.05)$.

Results: Most participants were female individuals (67.8\%), with five or more self-reported morbidities (62.6\%), without symptoms of depression (74.7\%) and $85.6 \%$ could perform basic activities of daily living independently. Functional independence in basic activities of daily living $(A D L)$ was associated with lack of symptoms of depression $(p=0.046)$ and instrumental activities of daily living (IADL) were associated with housing arrangement $(p=$ 0.045). Conclusion:Identification of the factors associated with the functional independence of elderlyindividuals aged 80 years or over contributes to improve the planning of nursing care for these individuals, in order to reduce or delay their dependence.
\end{abstract}

DESCRIPTORS: Elderly individuals aged 80 years or over; Longevity: Daily Activities; Geriatric Nursing.

\section{FACTORES ASOCIADOS A LA INDEPENDENCIA FUNCIONAL DE ANCIANOS LONGEVOS DE LA COMUNIDAD}

\author{
RESUMEN: \\ Objetivos: verificar la frecuencia de independencia funcional entre los longevos, así como los \\ factores que se asocian a la independencia funcional de ellos. \\ Metodología: estudio trasversal con 174 ancianos de 80 años y más, que viven en el área \\ urbana de Minas Gerais en el periodo de junio de 2017 a junio de 2018. Para elegir la \\ población, se utilizó el muestreo por conglomerado en múltiple estadio. Se realizaron los \\ análisis descriptivo, bivariado y de regresión logística múltiple $(p \leq 0,05)$. \\ Resultados: eran del sexo femenino $67,8 \%$, con cinco o más morbilidades auto referidas \\ $62,6 \%, 74,7 \%$ no presentaban síntomas depresivos y $85,6 \%$ eran independientes en las \\ actividades básicas de la vida diaria. La independencia funcional en las actividades básicas \\ de la vida diaria fue ausencia de indicativo de síntomas depresivos $(p=0,046)$ y las actividades \\ instrumentales de la vida cotidiana en la vivienda $(p=0,045)$. \\ Conclusión: la identificación de los factores asociados a la independencia funcional de los \\ ancianos ayudan el planeamiento del cuidado de enfermería que tiene como reto disminuir o \\ postergar la dependencia.
}

DESCRIPTORES: Anciano de 80 años o más; Longevidad; Actividades Cotidianas; Enfermería Geriátrica. 
Atualmente, os longevos, idosos com idade igual ou superior a 80 anos, representam $2,1 \%$ da população no Brasil e acredita-se que em 2060 esse percentual será de 8,4\%(1). No entanto, pouco se conhece acerca das condições de saúde dos longevos ${ }^{(2-4)}$.

A Organização Mundial da Saúde divulgou relatório que aborda a necessidade de mudança nas percepções de envelhecimento e saúde, pontuando, entre outros aspectos, que a idade avançada não significa dependência. Além disso, enfatiza-se a necessidade de alinhar os sistemas de saúde às demandas da população idosa, de acordo com a realidade de cada país, com objetivo único de maximizar a capacidade funcional(5).

Sob a ótica da saúde pública, a avaliação da capacidade funcional do idoso tornouse parâmetro essencial na prática da gerontologia ${ }^{(3)}$. Estudo entre idosos longevos acompanhados em uma Unidade Básica de Saúde do município de Curitiba-PR verificou que os profissionais de saúde, e especificamente os de enfermagem, precisam considerar a avaliação funcional como parte fundamental dos cuidados integrais aos idosos ${ }^{(2)}$.

Estudos nacional(3) e internacional(6) desenvolvidos entre idosos longevos da comunidade verificaram os fatores associados à independência funcional, e concluíram que ser aposentado $(p=0,007)$; praticar atividade física $(p<0,001)$; realizar atividade de lazer $(p<0,001)$; participar de grupos $(p=0,008)^{(3)}$; apresentar melhor desempenho físico $(p=0,043)$; autopercepção de saúde positiva $(p=0,020)$; ausência de fratura de quadril após os 55 anos de idade $(p=0,042)$ e de doença cardiovascular $(p=0,043)$ podem influenciar positivamente a independência funcional dos longevos ${ }^{(6)}$.

Ao considerar que fatores de risco e protetores diferem entre idosos jovens e longevos ${ }^{(7)}$ e que identificar precocemente os idosos com risco para incapacidade funcional possibilita aos profissionais de saúde o planejamento de intervenções com vistas à potencialização da autonomia e redução da dependência ${ }^{(8)}$, esse estudo teve como objetivos descrever as características sociodemográficas e clínicas de idosos longevos da comunidade; verificar a frequência de independência funcional entre esses idosos; e identificar os fatores associados à independência funcional desses idosos.

\section{MÉTODO}

Estudo de abordagem quantitativa, tipo inquérito domiciliar, transversal, observacional e analítico, que integra um projeto temático intitulado "Envelhecimento Ativo, Funcionalidade Global e Qualidade de Vida entre idosos da Microrregião de Saúde de Uberaba, Minas Gerais". Esta pesquisa desenvolveu-se na área urbana do município. Para a seleção da população utilizou-se a amostragem por conglomerado em múltiplo estágio.

Os critérios de inclusão considerados foram: ter 80 anos ou mais de idade e residir na zona urbana do município de Uberaba-MG. Foram excluídos os idosos hospitalizados e/ou institucionalizados; com problemas de comunicação como surdez, não corrigida por aparelhos, e transtornos graves da fala; com declínio cognitivo, avaliado pelo Mini exame do Estado Mental, seguindo os seguintes pontos de corte: $\leq 13$ para analfabetos, $\leq 18$ para escolaridade média (de um a 11 anos) e $\leq 26$ para alta escolaridade (superior a 11 anos) $)^{(9)}$.

Foram entrevistados 823 idosos, dentre os quais 15 apresentaram declínio cognitivo e 808 realizaram a entrevista completa, foram selecionados os idosos com 80 anos ou mais. Assim, a amostra do presente estudo foi composta por 174 idosos longevos.

A coleta dos dados foi realizada no domicílio dos idosos, de junho de 2017 a junho de 
2018, por meio de entrevista direta. Foram utilizados os instrumentos: Mini exame de Estado Mental(9); caracterização dos dados sociodemográficos por meio do formulário construído pelo Grupo de Pesquisa em Saúde Coletiva da Universidade Federal do Triângulo Mineiro (UFTM); Questionário Brasileiro de Avaliação Funcional e Multidimensional(10); Índice de Katz adaptado à realidade brasileira ${ }^{(11)}$; Escala de Lawton e Brody adaptada no Brasi( ${ }^{(12)}$; versão brasileira Short Physical Performance Battery(13) e a Escala de Depressão Geriátrica Abreviada ${ }^{(14)}$.

As variáveis do estudo foram: sexo (feminino; masculino); faixa etária (80-| 84 anos; 85 anos ou mais); arranjo de moradia (mora só; mora acompanhado(a)); número de morbidades autorreferidas (0-|4; 5 ou mais); indicativo de sintomas depressivos (sim; não); desempenho físico (incapacidade/baixo; moderado/bom); capacidade funcional para Atividades Básicas da Vida Diária (ABVD) (independente e dependente) e para Atividades Instrumentais da Vida Diária (AIVD) (independente e dependente total/parcial).

Para realizar as entrevistas, foram selecionados dez entrevistadores, os quais passaram por treinamento, capacitação e abordagem sobre questões éticas da pesquisa. Reuniões sistemáticas foram feitas entre os entrevistadores e pesquisadores, para acompanhamento e orientações da coleta dos dados. Após a coleta dos dados, foi elaborado um banco de dados em planilha do Excelß e realizada a dupla digitação. Posteriormente, foi efetuada a consistência entre as duas bases de dados e, quando necessário, procedeu-se à correção buscando o dado na entrevista original. Para a análise, o banco de dados foi importado para o programa Statistical Package for The Social Sciences (SPSS®), versão 22.0.

Os dados foram submetidos à análise descritiva e bivariada empregando-se razão de prevalência (RP), razão de chances de prevalência $(R C P)$ e o teste qui-quadrado $\left(x^{2}\right)$; e a regressão logística múltipla, tendo como desfecho a independência funcional. Foram consideradas como variáveis preditoras: sexo; arranjo de moradia; indicativo de sintomas depressivos; desempenho físico e número de morbidades autorreferidas. Este estudo considerou o intervalo de confiança de $95 \%$ e o nível de significância de $p \leq 0,05$.

O projeto foi aprovado pelo Comitê de Ética em Pesquisa com Seres Humanos da UFTM, com parecer de aprovação $n^{\circ} 2.053 .520$.

\section{RESULTADOS}

Do total de longevos entrevistados, verificou-se que a maioria era do sexo feminino $118(67,8 \%)$, com 85 anos ou mais de idade $89(51,1 \%)$, morava acompanhada $138(79,3 \%)$, com cinco ou mais morbidades autorreferidas $109(62,6 \%)$, sem indicativo de sintomas depressivos $130(74,7 \%)$ e com desempenho físico moderado/bom $97(55,7 \%)$, conforme Tabela 1.

Tabela 1 - Distribuição de frequência das variáveis sociodemográficas e clínicas de idosos longevos. Uberaba, MG, Brasil, 2018 (continua)

\begin{tabular}{lcc} 
Variáveis & $\mathbf{n}$ & $\%$ \\
\hline Sexo & & \\
\hline Feminino & 118 & 67,8 \\
\hline Masculino & 56 & 32,2 \\
\hline Faixa etária (em anos) & & \\
\hline $80-\mid$ 84 anos & 85 & 48,9 \\
\hline
\end{tabular}




\begin{tabular}{lcc}
\hline 85 ou mais & 89 & 51,1 \\
\hline Arranjo de moradia & & \\
\hline Mora acompanhado(a) & 138 & 79,3 \\
\hline Mora só & 36 & 20,7 \\
\hline Número de morbidades autorreferidas & & \\
\hline $0-\mid 4$ & 65 & 37,4 \\
\hline 5 ou mais & 109 & 62,6 \\
\hline Indicativo de sintomas depressivos & & \\
\hline Sem indicativo & 130 & 74,7 \\
\hline Com indicativo & 44 & 25,3 \\
\hline Desempenho físico & & \\
\hline Moderado/bom & 97 & 55,7 \\
\hline Incapacidade/baixo & 77 & 44,3
\end{tabular}

Quanto à frequência de independência funcional entre os idosos longevos, verificouse que 149 (85,6\%) eram independentes nas ABVD e $18(10,3 \%)$ nas AIVD.

O percentual foi maior para a independência funcional nas ABVD nos longevos do sexo feminino $102(86,4 \%)$, que morava só $33(91,7 \%)$, com menos de cinco morbidades autorreferidas $57(87,7 \%)$, sem indicativo de sintomas depressivos $116(89,2 \%)$ e com desempenho físico moderado/bom $88(90,7 \%)$, conforme Tabela 2.

Tabela 2 - Distribuição das variáveis sociodemográficas e clínicas de idosos longevos, de acordo com a independência funcional nas atividades básicas da vida diária. Uberaba, MG, Brasil, 2018 (continua)

\begin{tabular}{|c|c|c|c|c|c|c|c|c|c|}
\hline \multirow[t]{2}{*}{ Variáveis } & \multicolumn{2}{|c|}{$\begin{array}{l}\text { Independente } \\
\text { para as ABVD }\end{array}$} & \multicolumn{2}{|c|}{$\begin{array}{c}\text { Dependente } \\
\text { para as ABVD }\end{array}$} & \multirow[b]{2}{*}{$\mathbf{R P *}$} & \multirow[b]{2}{*}{$(\text { IC) })^{\star \star}$} & \multirow[b]{2}{*}{ RCP*** } & \multirow[b]{2}{*}{$(I C)^{\star \star}$} & \multirow[b]{2}{*}{$p^{\star \star \star \star}$} \\
\hline & $\mathbf{n}$ & $\%$ & $\mathbf{n}$ & $\%$ & & & & & \\
\hline \multicolumn{10}{|l|}{ Sexo } \\
\hline Feminino & 102 & 86,4 & 16 & 13,6 & 1,03 & $(0,90-1,17)$ & 1,22 & $(0,50-2,96)$ & 0,659 \\
\hline Masculino & 47 & 83,9 & 9 & 16,1 & & & & & \\
\hline \multicolumn{10}{|c|}{ Arranjo de moradia } \\
\hline $\begin{array}{l}\text { Mora } \\
\text { acompanhado(a) }\end{array}$ & 116 & 84,1 & 22 & 15,9 & 0,91 & $(0,81-1,03)$ & 0,47 & $(0,13-1,70)$ & 0,246 \\
\hline Mora só & 33 & 91,7 & 3 & 8,3 & & & & & \\
\hline \multicolumn{10}{|c|}{ Número de morbidades autorreferidas } \\
\hline $0-\mid 4$ & 57 & 87,7 & 8 & 12,3 & 1,03 & $(0,92-1,17)$ & 1,31 & $(0,53-3,24)$ & 0,55 \\
\hline 5 ou mais & 92 & 84,4 & 17 & 15,6 & & & & & \\
\hline \multicolumn{10}{|c|}{ Indicativo de sintomas depressivos } \\
\hline Não & 116 & 89,2 & 14 & 10,8 & 1,19 & $(0,99-1,42)$ & 2,76 & $(1,14-6,65)$ & 0,02 \\
\hline Sim & 33 & 75 & 11 & 25 & & & & & \\
\hline
\end{tabular}




\begin{tabular}{llllllllll}
\hline Desempenho físico & & & & & & & & & \\
\hline Moderado/bom & 88 & 90,7 & 9 & 9,3 & 1,14 & $(1-1,30)$ & 2,56 & $(1,06-6,18)$ & $\mathbf{0 , 0 3}$ \\
\hline $\begin{array}{l}\text { Incapacidade/ } \\
\text { baixo }\end{array}$ & 61 & 79,2 & 16 & 20,8 & & & & &
\end{tabular}

Legenda: *RP: Razão de prevalência; **IC: Intervalo de confiança; ${ }^{\star \star \star} R C P$ : OddsRatio; ${ }^{\star \star \star \star} p \leq 0,05$.

Destaca-se que o percentual foi maior para a independência funcional nas AIVD nos longevos do sexo masculino oito $(14,3 \%)$, que morava só sete $(19,4 \%)$, com menos de cinco morbidades autorreferidas nove $(13,8 \%)$, sem indicativo de sintomas depressivos 17 $(13,1 \%)$ e com desempenho físico moderado/bom 14 (14,4\%), conforme Tabela 3.

Tabela 3 - Distribuição das variáveis sociodemográficas e clínicas de idosos longevos, de acordo com a independência funcional nas atividades instrumentais da vida diária. Uberaba, MG, Brasil, 2018

\begin{tabular}{|c|c|c|c|c|c|c|c|c|c|}
\hline \multirow[t]{2}{*}{ Variáveis } & \multicolumn{2}{|c|}{$\begin{array}{l}\text { Independente } \\
\text { para AIVD }\end{array}$} & \multicolumn{2}{|c|}{$\begin{array}{c}\text { Dependente } \\
\text { para AIVD }\end{array}$} & \multirow[b]{2}{*}{ RP* } & \multirow[b]{2}{*}{$(\mathrm{IC})^{\star \star}$} & \multirow[b]{2}{*}{$\mathrm{RCP} * \star *$} & \multirow[b]{2}{*}{$(I C)^{\star \star}$} & \multirow[b]{2}{*}{$p^{* \star * *}$} \\
\hline & $\mathbf{n}$ & $\%$ & $\mathbf{n}$ & $\%$ & & & & & \\
\hline \multicolumn{10}{|l|}{ Sexo } \\
\hline Masculino & 8 & 14,3 & 48 & 85,7 & 0,59 & $(0,24-1,42)$ & 0,55 & $(0,20-1,49)$ & 0,24 \\
\hline Feminino & 10 & 8,5 & 108 & 91,5 & & & & & \\
\hline \multicolumn{10}{|l|}{ Arranjo de moradia } \\
\hline $\begin{array}{l}\text { Mora } \\
\text { acompanhado(a) }\end{array}$ & 11 & 8 & 127 & 92 & 0,41 & $(0,17-0,98)$ & 0,35 & $(0,12-1,00)$ & 0,044 \\
\hline Mora só & 7 & 19,4 & 29 & 80,6 & & & & & \\
\hline \multicolumn{10}{|c|}{ Número de morbidades autorreferidas } \\
\hline $0-\mid 4$ & 9 & 13,8 & 56 & 86,2 & 1,67 & $(0,70-4,00)$ & 1,78 & $(0,67-4,75)$ & 0,242 \\
\hline 5 ou mais & 9 & 8,3 & 100 & 91,7 & & & & & \\
\hline \multicolumn{10}{|c|}{ Indicativo de sintomas depressivos } \\
\hline Não & 17 & 13,1 & 113 & 86,9 & 5,75 & $\begin{array}{l}(0,78- \\
41,99) \\
\end{array}$ & 6,46 & $\begin{array}{l}(0,83- \\
50,10) \\
\end{array}$ & 0,042 \\
\hline Sim & 1 & 2,3 & 43 & 97,7 & & & & & \\
\hline \multicolumn{10}{|l|}{ Desempenho físico } \\
\hline Moderado/bom & 14 & 14,4 & 83 & 85,6 & 2,77 & $(0,95-8,10)$ & 3,07 & $(0,97-9,76)$ & 0,047 \\
\hline $\begin{array}{l}\text { Incapacidade/ } \\
\text { baixo }\end{array}$ & 4 & 5,2 & 73 & 94,8 & & & & & \\
\hline
\end{tabular}

Legenda: *RP: Razão de prevalência; ${ }^{\star \star \mid C: ~ I n t e r v a l o ~ d e ~ c o n f i a n c ̧ a ; ~}{ }^{\star \star \star R C P: ~ O d d s R a t i o ; ~}{ }^{\star \star \star \star ~} \mathrm{p} \leq 0,05$.

A independência funcional nas ABVD associou-se à ausência de indicativo de sintomas depressivos $(p=0,046)$, conforme Tabela 4. Já a independência funcional para as AIVD associou-se à variável morar acompanhado, $(p=0,045)$, conforme Tabela 5. 
Tabela 4 - Modelo final de regressão logística múltipla para as variáveis associadas à independência funcional nas atividades básicas da vida diária de idosos longevos. Uberaba, MG, Brasil, 2018

\begin{tabular}{lccl} 
Variáveis & $\mathbf{R C P}$ & $(\mathbf{I C})^{\star *}$ & $\mathbf{p}^{\boldsymbol{*}}$ \\
\hline Sexo & 0,55 & $(0,21-1,46)$ & 0,233 \\
\hline Arranjo de moradia & 2,62 & $(0,69-9,85)$ & 0,153 \\
\hline Número de morbidades autorreferidas & 0,8 & $(0,30-2,09)$ & 0,653 \\
\hline Indicativo de sintomas depressivos & 0,38 & $(0,15-0,98)$ & $\mathbf{0 , 0 4 6}$ \\
\hline Desempenho físico & 0,39 & $(0,15-1,00)$ & 0,052
\end{tabular}

Legenda: *RCP: OddsRatio; ${ }^{* \star} I C$ : Intervalo de confiança; ${ }^{* \star \star} p \leq 0,05$.

Tabela 5 - Modelo final de regressão logística múltipla para as variáveis associadas à independência funcional nas atividades instrumentais da vida diária de idosos longevos. Uberaba, MG, Brasil, 2018

\begin{tabular}{lccc} 
Variáveis & $\mathbf{R C P}$ & $(\mathbf{I C})^{\star *}$ & $\mathbf{p}^{\boldsymbol{*}}$ \\
\hline Sexo & 1,39 & $(0,48-3,98)$ & 0,54 \\
\hline Arranjo de moradia & 3,01 & $(1,02-8,82)$ & $\mathbf{0 , 0 4 5}$ \\
\hline Número de morbidades autorreferidas & 0,72 & $(0,25-2,04)$ & 0,541 \\
\hline Indicativo de sintomas depressivos & 0,18 & $(0,22-1,50)$ & 0,114 \\
\hline Desempenho físico & 0,42 & $(0,12-1,41)$ & 0,162
\end{tabular}

Legenda: ${ }^{*}$ RCP: OddsRatio; ${ }^{* \star} \mid C$ : Intervalo de confiança; ${ }^{* \star *} p \leq 0,05$.

O maior percentual de idosos longevos do sexo feminino corrobora com estudos nacional(15-16) e internacional ${ }^{(17)}$ desenvolvidos na comunidade. As mulheres apresentam maior longevidade em relação aos homens, fato que pode estar associado aos aspectos sociais e ao comportamento individual em relação ao cuidado com a própria saúde ${ }^{(18)}$. Os homens estão mais expostos aos riscos de acidentes de trabalho, à violência e aos hábitos de vida não saudáveis, como o tabagismo e o etilismo, enquanto as mulheres procuram os serviços de saúde e aderem aos programas de prevenção com maior frequência ${ }^{(19)}$.

Quanto à faixa etária, resultados divergentes foram identificados em estudos nacionais desenvolvidos entre idosos longevos, os quais a maioria apresentava entre 80 a 84 anos de idade ${ }^{(15)}$. Ao ampliar a quantidade de anos vividos, é necessário que os serviços de saúde desenvolvam estratégias voltadas à promoção da saúde e à prevenção de agravos, proporcionando a esses indivíduos independência funcional pelo período de tempo mais longo possível ${ }^{(3)}$.

Os resultados do arranjo de moradia foram semelhantes ao inquérito entre idosos longevos da comunidade em Recife-PE, o qual verificou que 38 (88\%) moravam acompanhados ${ }^{(18)}$. Entretanto, em estudos internacionais houve predomínio de idosos longevos que moravam sós, sendo $380(95,7 \%)$ na Suécia ${ }^{(20)}$ e $404(60,6 \%)$ no Reino Unido(17). Acredita-se que idosos que se sentem responsáveis pela sua sobrevivência e da família, que se preocupam com o cuidado do parceiro e que mantém vida social ativa, tendem a 
desenvolver mais atividades diárias e serem mais ativos ${ }^{(21)}$.

O número de morbidades autorreferidas foi semelhante ao identificado nos estudos nacionais ${ }^{(4,15-16)}$ e internacional(17) entre idosos longevos da comunidade, os quais a maioria apresentava cinco ou mais morbidades. As doenças crônicas não transmissíveis tornam-se mais frequentes com o avançar da idade e possuem relação direta com a independência dos idosos, influenciando na realização das Atividades da Vida Diária (AVD) (15). Por isso, a prevenção dessas condições mediante o suporte necessário para cada indivíduo deve ser o objetivo principal dos sistemas de saúde.

Em relação ao indicativo de presença de sintomas depressivos, estudos nacionais com idosos longevos da comunidade obtiveram resultados similares à presente pesquisa: a maioria não apresentou indicativo de sintomas depressivos ${ }^{(22)}$. A presença de depressão é, hoje, considerada importante preditor de incapacidade, com desencadeamento e agravamento do declínio funcional ${ }^{(23)}$. Assim, há de salientar a necessidade dos profissionais de saúde, com destaque a equipe de enfermagem, de reconhecer a sintomatologia depressiva entre os idosos, pois a doença ainda é subdiagnosticada e, muitas vezes, considerada como manifestações naturais consequentes do envelhecer ${ }^{(15)}$.

Sobre o desempenho físico, resultados semelhantes ao presente estudo foram verificados na pesquisa internacional desenvolvida entre idosos longevos residentes no Sudoeste dos Estados Unidos, na qual 750 (54,9\%) apresentaram desempenho físico moderado/bom ${ }^{(24)}$. Sabe-se que o baixo desempenho físico, dificuldades nas AVD e o sedentarismo são preditores independentes de mortalidade, mesmo na ausência de doenças agravantes ${ }^{(25)}$. Desta forma, a avaliação do desempenho físico em idosos longevos deve ser introduzida nos atendimentos dos profissionais de saúde para que as mudanças sejam acompanhadas no decorrer dos anos e traçados planos de intervenções ${ }^{(25)}$.

Considerar questões ligadas à realidade dos idosos longevos, para identificar os fatores associados à sua independência funcional, possibilita a elaboração e o planejamento de ações de saúde direcionadas às necessidades específicas desse grupo(15).

Concernente à prevalência de independência funcional para ABVD (149 - 85,6\%) entre idosos longevos, percentuais inferiores foram identificados em investigações nacionais realizadas em Jequié-BA $69(59,0 \%)^{(15)}$ e Lafaiete Coutinho-BA 33 (75,3\%) $)^{(22)}$; e em estudo internacional na Suécia $98(25 \%)^{(26)}$.

Em relação à independência funcional para AIVD, resultados superiores aos do presente estudo foram identificados em investigações nacionais entre idosos longevos da comunidade ${ }^{(15,22)}$.

Nesse contexto, cabe destacar a escassez de estudos, no âmbito nacional e internacional, relacionados à independência em AVD de idosos longevos ${ }^{(18,27)}$. Isso é corroborado em revisão sistemática com metanálise entre estudos com idosos brasileiros, na qual a capacidade funcional entre aqueles com idade avançada foi avaliada em apenas duas $(8,7 \%)$ das pesquisas ${ }^{(27)}$.

O elevado percentual de idosos independentes nas atividades de autocuidado sugere que idosos longevos estão cada vez mais permanecendo em suas atividades sociais e participativas, favorecendo sua mobilidade e independência ${ }^{(21)}$.

A menor prevalência de independência para AIVD quando comparados com as ABVD pode ocorrer devido à maior exigência de integridade física e cognitiva para que aquelas sejam realizadas ${ }^{(15,28)}$; pela perda hierárquica de atividades mais complexas ${ }^{(28)}$; da insegurança do idoso na realização de atividades que estão relacionadas às habilidades mais complexas; e familiares que não permitem determinadas atividades devido ao avanço da idade ${ }^{(29)}$.

Logo, é importante que políticas públicas sejam baseadas de acordo com o perfil da população a qual será vinculada ${ }^{(18)}$. No que concerne à capacidade funcional, há maior 
possibilidade de identificação precoce daqueles em maior risco para dependência; e para melhora ou estabilidade da independência com o avanço da idade ${ }^{(3,22)}$. Assim, o objetivo de intervenções e estratégias de promoção da saúde é pautado na manutenção da capacidade funcional para que a autonomia e independência sejam mantidas ${ }^{(22)}$.

Em relação aos fatores associados, investigação internacional realizada na Suécia identificou associação entre dependência nas atividades de autocuidado, como transferência $(p=0,007)$ e vestir-se $(p=0,035)$, e $o$ indicativo de sintomatologia depressiva ${ }^{(26)}$. Apesar da relação desse estudo ter sido inversa à da presente pesquisa, é possível que idosos sem indicativo de depressão mantenham com maior facilidade as ABVD, já que há menor probabilidade de efeitos deletérios de sintomas do indicativo de depressão, como fadiga, diminuição de energia, perda de apetite e peso, estarem presentes ${ }^{(26)}$.

Destaca-se que a maioria dos estudos analisam possíveis fatores de risco para a dependência funcional, o que dificulta a comparação entre os resultados ${ }^{(4,7,15,22,26)}$. Ainda, é importante compreender que as variáveis relacionadas à capacidade funcional podem diferir entre faixas etárias do grupo de idosos ${ }^{(7)}$.

Em revisão sistemática internacional, idosos com companheiro(a) $(p<0,001)$ apresentaram melhor capacidade funcional, sendo essa variável considerada um fator de proteção para as atividades de vida diária(7), o que condiz com dados do presente estudo. Além disso, a coabitação pode ser considerada estratégia que beneficia o idoso(30) e idosos que moram acompanhados podem apresentar fortalecimento e incentivo ${ }^{(18)}$, principalmente para a realização de atividades.

Assim, a identificação dos fatores associados à independência funcional de idosos longevos auxilia na determinação de plano de cuidados individualizado, direcionado às suas reais necessidades e características e capaz de potencializar a manutenção da sua independência funcional.

O delineamento transversal do estudo constitui uma limitação que não permite determinar as relações de causalidade e estabelecer medidas de proteção para a independência funcional.

\section{CONCLUSÃO}

No presente estudo, houve predomínio de longevos do sexo feminino, com 85 anos ou mais de idade, que moravam acompanhados, com cinco ou mais morbidades autorreferidas, sem indicativo de sintomas depressivos e com desempenho físico moderado/bom.

Dos idosos investigados, $85,6 \%$ foram considerados independentes nas ABVD e $10,3 \%$ nas AIVD. O fator significativamente associado à independência funcional nas ABVD foi ausência de indicativo de sintomas depressivos, enquanto que, nas AIVD, foi o arranjo de moradia.

O conhecimento relativo à capacidade funcional favorece que os profissionais de saúde avaliem a necessidade de auxílio dos idosos longevos para atividades de manutenção e promoção da própria saúde, bem como desenvolvam ações em busca da sua potencialização, possibilitando assim que o idoso viva da forma mais independente possível.

\section{REFERÊNCIAS}


Disponível em: https://biblioteca.ibge.gov.br/visualizacao/livros/liv98965.pdf.

2. Pereira LF, Lenardt MH, Michel T, Carneiro NHK, Bento LF. Retrato do perfil de saúde-doença de idosos longevos usuários da atenção básica de saúde. Rev enferm UERJ [Internet]. 2015 [acesso em 10 jul 2018]; 23(5). Disponível em: https://doi.org/10.12957/reuerj.2015.5069.

3. Ribeiro DKMN, Lenardt MH, Michel T, Setoguchi LS, Grden CRB, Oliveira ES de. Fatores contributivos para a independência funcional de idosos longevos. Rev Esc Enferm USP. [Internet]. 2015 [acesso em 10 mar 2018]; 49(1). Disponível em: http://dx.doi.org/10.1590/S0080-623420150000100012.

4. Bortoluzzi EC, Doring M, Portella MR, Cavalcanti G, Mascarelo A, Delani MP. Prevalência e fatores associados a dependência funcional em idosos longevos. Rev. bras. ativ. fís. saúde [Internet]. 2017 [acesso em 20 jul 2018]; 22(1). Disponível em: https://doi.org/10.12820/rbafs.v.22n1p85-94.

5. Organização Mundial de Saúde (OMS). Relatório Mundial de Envelhecimento e Saúde. Genebra: Organização Mundial de Saúde; 2015.

6. Vaughan L, Leng X, La Monte MJ, Tindle HA, Cochrane BB, Shumaker SA. Functional Independence in Late-Life: Maintaining Physical Functioning in Older Adulthood Predicts Daily Life Function after Age 80. J Gerontol [Internet]. 2016 [acesso em 01 mar 2018]; 71(1). Disponível em: https://doi.org/10.1093/gerona/ glv061.

7. Van der Vorst A, Zijlstra GA, Witte N, Duppen D, Stuck AE, Kempen Gl et al. Limitations in Activities of Daily Living in Community-Dwelling People Aged 75 and Over: A SystematicLiterature Review of RiskandProtectiveFactors. PloS One. [Internet]. 2016 [acesso em 13 set 2018]; 11(10). Disponível em: https://doi.org/10.1371/journal.pone.0165127.

8. Souza F, Dias AM. Condição multidimensional de saúde dos idosos inscritos na estratégia saúde da família. Arq ciênc. saúde [Internet]. 2015 [acesso em 10 set 2018]; 22(4). Disponível em: https://doi. org/10.17696/2318-3691.22.4.2015.157.

9. Bertolucci PHF, Brucki SMD, Campacci SR, Juliano Y. O Mini-Exame do Estado Mental em uma população geral: impacto da escolaridade. Arq. Neuro-Psiquiatr. [Internet]. 1994 [acesso em 10 mar 2018]; 52(1). Disponível em: http://dx.doi.org/10.1590/S0004-282X1994000100001.

10. Ramos LR, Perracini M, RosaTE.Kalache A. Significance and management of disability among urban elderly residents in Brazil. J Cross Cult Gerontol. [Internet]. 1993 [acesso em 10 mar 2018]; 8(4). Disponível em: http://dx.doi.org/10.1007/BF00972560.

11. Lino VTS, Pereira SRM, Camacho LAB, Ribeiro Filho ST,Buksman S. Cross-cultural adaptation of the Independence in Activities of Daily Living Index (Katz Index). Cad. Saúde Pública. [Internet]. 2008 [acesso em 10 mar 2018]; 24(1). Disponível em: http://dx.doi.org/10.1590/S0102-311X2008000100010.

12. Santos RL dos, Virtuosos Júnior JS. Confiabilidade da versão brasileira da escala de atividades instrumentais da vida diária. Rev. bras. promoç. saúde [Internet]. 2012 [acesso em 10 mar 2018]; 21(4). Disponível em: http://dx.doi.org/10.5020/18061230.2008.p290.

13. Nakano MM. Versão brasileira da Short Physical Performance Battery SPPB : adaptação cultural e estudo da confiabilidade [dissertação]. Campinas (SP): Faculdade de Educação, Universidade Estadual de Campinas; 2007. 163 p. Disponível em: http://repositorio.unicamp.br/jspui/handle/REPOSIP/252485.

14. Almeida OP, Almeida SA. Confiabilidade da versão brasileira da Escala de Depressão em Geriatria (GDS) versão reduzida. Arq. Neuro-Psiquiatr. [Internet]. 1999 [acesso 20 mar 2018]; 57(2). Disponível em: http://dx.doi.org/10.1590/S0004-282X1999000300013.

15. Novais MM, Araújo CM, Bôas SV, Prates RV, Pinto DS, Reis LA. Avaliação de indicadores de desempenho funcional de idosos longevos residentes em domicílio. Arq ciênc. saúde [Internet]. 2016 [acesso 21 ago 2018]; 23(3). Disponível em: https://doi.org/10.17696/2318-3691.23.3.2016.280.

16. Queiroz DB, Araújo CM de, Oliveira LC de, Novais MM, Andrade LA, Reis LA. Funcionalidade, aptidão motora e condições de saúdeem idosos longevos residentes em domicílio. Arq ciênc. saúde [Internet]. 
2016 [acesso 10 ago 2018]; 23(2). Disponível em: https://doi.org/10.17696/2318-3691.23.2.2016.281.

17. Collerton, Jagger C, Yadegarfar ME, Davies K, Parker SG, Robinson L et al. Deconstructing Complex Multimorbidity in the Very Old: Findings from the Newcastle 85+ Study. Biomed Res Int. [Internet]. 2016 [acesso 13 set 2018]. Disponível em: http://dx.doi.org/10.1155/2016/8745670.

18. Porciuncula RCR da, Carvalho EF de, BarretoKML, Leite VMM. Perfil socioepidemiológico e autonomia de longevos em Recife-PE, Nordeste do Brasil. Rev. Bras. Geriatr. Gerontol. [Internet]. 2014 [acesso em 28 jul 2018]; 17(2). Disponível em: http://dx.doi.org/10.1590/S1809-98232014000200009.

19. Faustino AM, Moura LBA, Gandolfi L. Relação entre violência e função cognitiva em idosos. Rev enferm UFPE. [Internet]. 2016 [acesso 09 jun 2018]; 10(5). Disponível em: http://dx.doi.org/10.5205/ reuol.9003-78704-1-SM.1005201618.

20. Ekstrom H, Schmidt SM, Iwarsson S. Home and health among different sub-groups of the ageing population: a comparison of two cohorts living in ordinary housing in Sweden. BMC Geriatr [Internet]. 2016 [acesso 13 set 2018]; 16(90). Disponível em: http://dx.doi.org/10.1186/s12877-016-0265-7.

21. Lenardt MH, Grden CRB, Sousa JAV, Betiolli SE, Reche RM, Lourenço TM. Fatores sociodemográficos e clínicos associados à força de preensão manual e velocidade da marcha em longevos. Cogitare enferm. [Internet]. 2017 [acesso 21 nov 2018]; 22(3). Disponível em: http://dx.doi.org/10.5380/ce.v22i3.50464.

22. Brito TA, Fernandes MH, Coqueiro RS, Jesus CS, Freitas R. Functional capacity and associated factors among longevous senior individuals living in community: a population study in Northeastern Brazil. Fisioter. Pesqui. [Internet]. 2014 [acesso em 09 set 2018]; 21(4). Disponível em: http://www.scielo.br/ scielo.php?pid=S1809-29502014000400308\&script=sci abstract.

23. Almeida MASO, Lemes AG, Nascimento VF, Fonseca PIMN, Rocha HAO et al. Fatores de risco associados à depressão em idosos no interior de Mato Grosso. Rev. baiana saúde pública [internet]. 2015 [acesso 15 jun 2018]; 39(3). Disponível em: http://dx.doi.org/10.5327/Z0100-0233-2015390300012.

24. Panas LJ, Siordia C, Angel RJ, Eschbach K, Markides KS. Physical performance and short-term mortality in very old mexicanamericans. Exp Aging Res. [Internet]. 2013 [acesso 22 ago 2018]; 39(5). Disponível em: https://dx.doi.org/10.1080/0361073X.2013.839021.

25. Nascimento JS, Tavares DMS. Prevalência e fatores associados a quedas em idosos. Texto contexto - enferm. [Internet]. 2016 [acesso 13 set 2018]; 25(2). Disponível em: http://dx.doi.org/10.1590/0104$\underline{07072016000360015 .}$

26. Boström G, Conradsson M, Rosendahl E, Nordström P, Gustafson Y, Littbrand H. Functional capacity and dependency in transfer and dressing are associated with depressive symptoms in older people. Clin Interv Aging [Internet]. 2014 [acesso 10 set 2018]; 2014(9). Disponível em: https://dx.doi.org/10.2147/ CIA.S57535.

27. Campos ACV, Almeida MHM de, Campos GV, Bogutchi TF. Prevalência de incapacidade funcional por gênero em idosos brasileiros: uma revisão sistemática com metanálise. Rev. Bras. Geriatr.

Gerontol. [Internet]. 2016 [acesso 30 ago 2018]; 19(3). Disponível em: http://dx.doi.org/10.1590/180998232016019.150086.

28. Freitas RS, Fernandes MH, Coqueiro RS, Reis Júnior WM, Rocha SV, Brito TA. Capacidade funcional e fatores associados em idosos: estudo populacional. Acta Paul. Enferm.[Internet]. 2012 [acesso 14 ago 2018]; 25(6). Disponível em: https://dx.doi.org/10.1590/S0103-21002012000600017.

29. Pinto DS, Novais MM, Prates RV, Bôas SV, Araújo CM de, Reis LA. Atividades funcionais e nível de dependência em idosos longevos residentes em domicílio. Rev Pesqui. Fisioter. [Internet]. 2017 [acesso 13 set 2018]; 7(3). Disponível em: http://dx.doi.org/10.17267/2238-2704rpf.v7i3.1500.

30. Reis LA, Torres GV, Xavier TT, Silva RAR, Costa IKF, Mendes FRP. Percepção do suporte familiar em idosos de baixa renda e fatores associados. Texto contexto- enferm. [Internet]. 2011 [acesso 02 set 2018]; 20(n.esp). Disponível em: http://dx.doi.org/10.1590/S0104-07072011000500006. 
Recebido: $13 / 09 / 2018$

Finalizado: 30/04/2019

Autor Correspondente:

Darlene Mara dos Santos Tavares

Universidade Federal do Triângulo Mineiro

Av. Getúlio Guaritá, 159 - 38025-440 - Uberaba, MG, Brasil

E-mail: darlene.tavares@uftm.edu.br

\section{Contribuição dos autores:}

Contribuições substanciais para a concepção ou desenho do estudo; ou a aquisição, análise ou interpretação de dados do estudo - DMST, NCG, LALS, GFM

Elaboração e revisão crítica do conteúdo intelectual do estudo - DMST, NCG, LALS, GFM

Aprovação da versão final do estudo a ser publicado - DMST, NCG, LALS, GFM

Responsável por todos os aspectos do estudo, assegurando as questões de precisão ou integridade de qualquer parte do estudo - DMST, NCG, LALS, GFM 